\title{
Restauration d'une passe et des fonds de la lagune de Goro (Delta du Po, Italie)
}

\author{
Umberto Simeoni, Corinne Corbau, \\ Dipartimento di Scienze della Terra - Università degli Studi di Ferrara \\ Via Saragat 1, 44100 Ferrara, Italia \\ g23@unife.it,.cbc@unife.it
}

\section{Résumé}

La Sacca di Goro, située dans le Parc Régional du Delta du Po, s'étend sur 2600 ha et, est caractérisée par une forte pression anthropique. Dans ce contexte, une intervention, réalisée au niveau d'une passe lagunaire d'origine anthropique, vise à améliorer l'échange des masses d'eau entre la lagune et la mer et assainir les fonds de la lagune afin d'améliorer la productivité de l'activité de pêche.

Ce projet représente un bon compromis entre les exigences socio-économiques de la zone et les solutions techniques disponibles. Il prévoit le re-profilage de la plate-forme submergée de la flèche littorale et du canal de marée et également le rechargement de l'île-barrière pour protéger les territoires lagunaires. Les sédiments plus fins seront utilisés pour assainir les fonds lagunaires.

\begin{abstract}
The Sacca di Goro, located in the Regional Parc of the Po Delta, extends over 2600 ha and, is characterised by an intense human pressure. In this context, an intervention, realised at the second mouth of the lagoon (anthropical), aims to improve the exchange of waters between the lagoon and the sea and to reclaim the sea floor of the lagoon in order to improve the production of the fishing activities.

The project represents a good compromise between the socio-economical needs of the area and the available technical solutions. It anticipates the re-profilage of the submerged platform of the littoral spit and of the tidal channel but also the nourishment of the barrier-island in order to put in security the lagoonal territories. The finer sediments will be deposited on the lagoon floor.
\end{abstract}




\section{Mots-clés}

Gestion intégrée, lagune, passe lagunaire, hydrodynamique, évolution géomorphologique, Goro, Italie

\section{Introduction}

Les lagunes côtières correspondent à une zone de transition entre le milieu terrestre et le milieu marin recouvert d'une nappe d'eau peu profonde, douce, salée ou saumâtre (KJERFVE, 1994). L'intérêt pour les milieux lagunaires, qui occupent environ $13 \%$ des côtes mondiales, et de leurs structures sédimentaires augmente de manière considérable notamment du point de vue de leur conservation. De nombreuses études concernent leur aspects hydrologiques, biologiques et écologiques (BARNES, 1980) mais au cours de ces dernières années l'intérêt se porte sur les fonctions écologiques et biogéochimiques (CAUMETTE et al, 1996 ; GIORDANI et al., 2005).

Ces milieux présentent une dynamique évolutive très complexe et rapide liée aux agents et aux flux de matériel entre le milieu terrestre, marin et atmosphérique (BARNES, 1980). Les échanges hydriques entres les lagunes et la mer sont réglés par les passes tidales qui interrompent la dérive littorale en influençant ainsi l'apport de sable des plages adjacentes.

L'organisation morphodynamique et l'efficacité des passes naturelles peuvent se transformer en quelques années, et notamment en raison de l'activité humaine. Pour rétablir leur efficience des dragages visant au reprofilage des passes sont parfois réalisés. Cette opération, si réalisée correctement, permet de maintenir la puissance hydraulique des embouchures tidales et de disposer de matériels pour des interventions de rechargement artificiel. Par exemple TSIHRINTZIZ et al. (2007) ont proposés différentes solutions d'intervention pour améliorer les échanges d'eau au niveau de la lagune de Vassova (Grèce). Dans la zone Nord Adriatique, un système de lagunes se développe sur environ $200 \mathrm{~km}$, depuis l'embouchure de fleuve Isonzo jusqu'à Comacchio.

\section{Sacca di Goro}

La Sacca di Goro actuelle (Fig. 1) est caractérisée par une interaction diachronique entre les composantes marines et terrestres et aussi par une intense action anthropique contemporaine. 
La lagune s'étend sur environ 2600 ha pour un volume d'eau d'environ 39,5 millions de $\mathrm{m}^{3}$, et la profondeur moyenne des fonds est d'environ $0,6 \mathrm{~m}$ (GABBIANELLI et al., 2000). La Sacca est également un milieu de forte valeur environnementale: elle est classée comme «zone humide d'importance internationale » selon la Convention Ramsar, comme réserve naturelle pour le peuplement animal et est comprise dans le Parc Régional du Delta du Po de l'Emilia-Romagna. De plus elle possède une forte valeur économique pour les activités de pêche et de culture de coque employant plus de 1000 personnes.

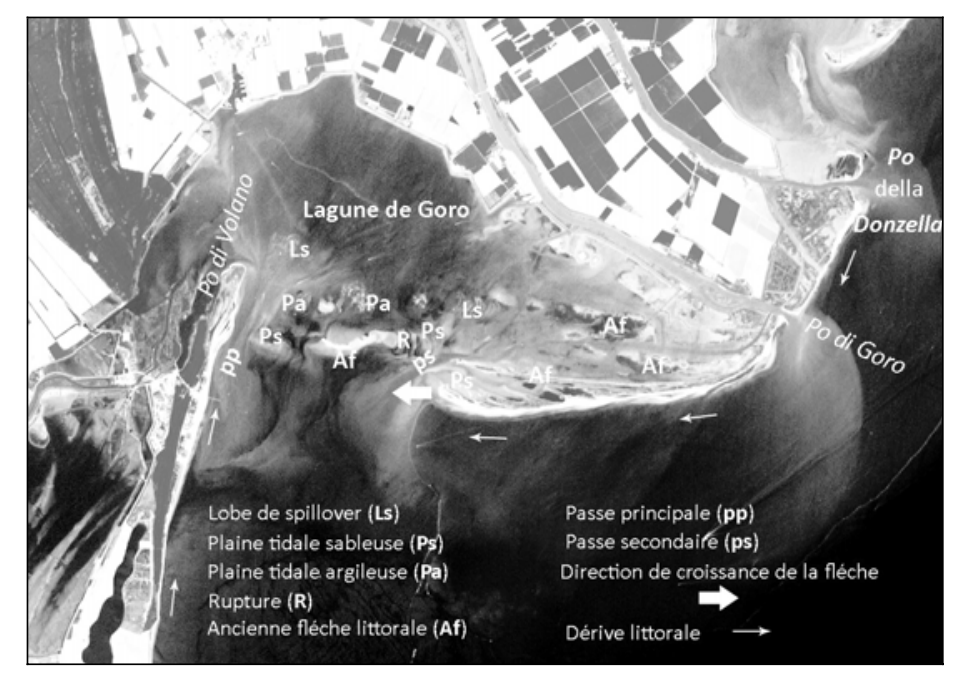

Figure 1 - Photo satellitaire de la Sacca di Goro montrant les principales morphologies de la passe lagunaire

Les échanges hydriques avec la mer se réalisent au niveau de la passe principale (Ouest) et celle secondaire (Est) d'origine anthropique qui s'est développée suite à l'élargissement du canal artificiel réalisé de manière abusive en 1991. Le flot pénètre à l'intérieur de la lagune d'abord au niveau de la passe secondaire et, ensuite, au niveau de la passe principale. Il convient de mentionner que différentes interventions ont été effectuées pour améliorer les échanges hydriques.

\section{Morphodynamique de la passe secondaire}

Sur une période de 20 ans la passe secondaire de la Sacca de Goro est passée d'un canal artificiel large de quelques dizaines de mètres à une ouverture tidale bien structurée et large de plus de 1,5 km (Fig. 1). Le canal a été creusé dans une 
zone d'élargissement des directions du transport sédimentaire (zone d'ombre) et donc de faible alimentation liée aux effets de diffraction de la houle à l'intérieur du passage qui ont conduit à une érosion progressive des rives. Au cours de la même période, la croissance de la flèche littorale vers Volano (GABBIANELLI et al., 2000) a entrainé une réduction de la section de la passe principale. Le nouveau canal artificiel a pris en charge une partie du prisme tidal du bassin lagunaire, donnant naissance à un processus de formation d'une nouvelle passe tidale.

Les premières formes identifiables du système tidal apparaissent en 1995. L'action de la houle, des courants tidaux et de la dérive littorale contrôle l'évolution morphologique de la passe et a engendré la croissance de nouvelle ramification de la flèche.

Le delta de jusant délimité au niveau de sa partie terminale par l'isobathe $2 \mathrm{~m}$, correspond à la surélévation morphologique typique du lobe terminal, comme mis en évidence par la sinuosité des isobathes 2,3-3 m. De plus la dérive littorale, orientée vers l'ouest, dérive le jusant vers le large et à l'ouest de la passe en favorisant un développement majeur du lobe gauche du delta de jusant. Les faibles courants tidaux ont déterminé le dépôt des matériaux à l'intérieur du canal tidal en le forçant à une migration compensatoire. De plus la structure du système deltaïque détermine une séparation complète des flux tidaux.

\section{Evolution récente de la flèche litorale (Scanno di Goro)}

La comparaison des relevés bathymétriques de Mai 2003 et Septembre 2005 a permis de calculer les variations volumétriques des fonds de l'isobathe $0,5 \mathrm{~m}$ à 4 $\mathrm{m}$ représentant une superficie de $5.163 .000 \mathrm{~m}^{2}$ environ (SIMEONI et BRUNELLI, 2007). Le budget total sédimentaire 2003-2005 apparaît positif avec une augmentation d'environ $1.623 .000 \mathrm{~m}^{3}$, qui correspond à un dépôt d'environ 0,31 m d'épaisseur.

Une étude plus détaillée, utilisant la même procédure et basée sur cinq relevés bathymétriques, a été réalisée entre Mai 2003 et Décembre 2006 pour la zone orientale de la passe secondaire sur une superficie de $1.045 .000 \mathrm{~m}^{2}$. L'évolution des fonds de la passe lagunaire entre Mai 2003 et Décembre 2006 (32 mois) est positive (Fig. 2) et représente une augmentation des volumes sédimentaires de $585.000 \mathrm{~m}^{3}$ environ, équivalant à une épaisseur de $0,56 \mathrm{~m}$.

La comparaison des bathymétries de Janvier et Décembre 2006 indique une augmentation volumétrique de $161.800 \mathrm{~m}^{3}$ de matériel. Une zone correspondant 
au canal tidal présente un abaissement des fonds lié aux interventions de dragages réalisées au cours de la même période. Les comparaisons bathymétriques indiquent un apport annuel de matériel de $160.000 \mathrm{~m}^{3}$.

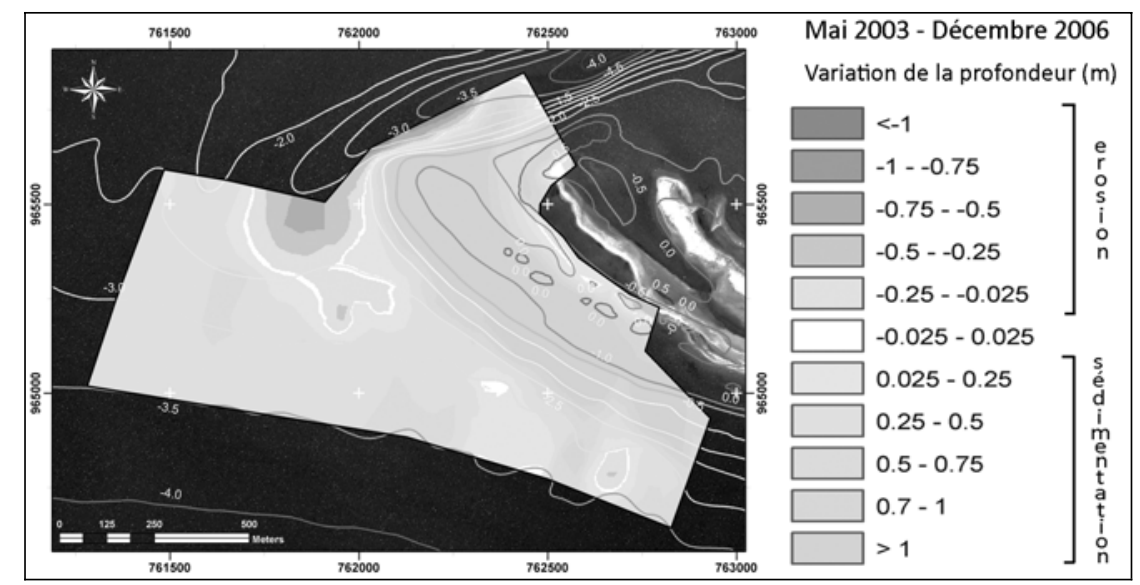

Figure 2 - Variations des fonds marins obtenues par comparaison des relevés bathymétriques de Mai 2003 et Décembre 2006.

Ces données sont toutefois sous-estimées en raison de l'absence de données relative à la partie émergée du spit et des petits fonds compris 0 et $0,5 \mathrm{~m}$. En effet l'observation des photos satellitaires de Mai 2003 et Juillet 2007 (Fig. 3) et les relevés topographiques de la zone montrent que la partie terminale émergée du spit se soit développée de manière importante.

Le calcul planimétrique indique un gain $10.500 \mathrm{~m}^{2}$ entre Mai 2003 et Août 2004, une perte d'environ $2.000 \mathrm{~m}^{2}$ entre Août 2004 et Septembre 2005, un autre gain de $10.600 \mathrm{~m}^{2}$ entre Septembre 2005 et Décembre 2006 et un dernier gain de $97.600 \mathrm{~m}^{2}$ entre Décembre 2006 et Juillet 2007. Dans l'ensemble le budget de 2003 à 2007 est positif avec une augmentation de la superficie de $117.000 \mathrm{~m}^{2}$ environ.

Bien que l'évaluation obtenue avec ces comparaisons n'ait qu'une valeur semi quantitative, le bilan résulte positif. Ces données témoignent du fort dynamisme de la zone caractérisé par l'apparition et le démantèlement de vaste superficie par rapport à toute la superficie de plage émergée considérée. 


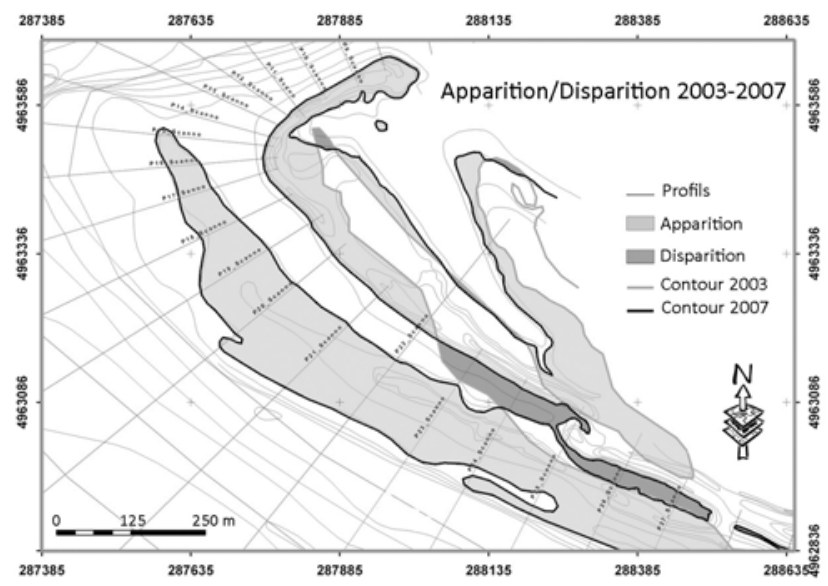

Figure 3 -Représentation des zones émergées apparues et disparues entre 2003 et 2007.

\section{Intervention pour l'aménagement de la passe tidale}

Depuis l'ouverture de la passe lagunaire secondaire, le spit a toujours eu un fort développement comme l'indique la vaste plate-forme d'alimentation, avec un front de progradation situé au niveau de l'isobathe $2.5 \mathrm{~m}$, et sa prolongation vers l'ouest (SIMEONI et al., 2007b). De plus, la progradation liée au processus de croissance de la flèche interagit sur la géométrie du canal tidal en induisant sa rotation vers l'ouest par rapport à la perpendiculaire aux sections de la passe.

Un excès de sédimentation s'observe sur les fonds à l'extrémité de la flèche sableuse au détriment de ceux de l'île barrière en raison du déplacement vers la mer du côté amont de la passe par rapport à celui aval. Ce phénomène peut être dû au concept de flux local inverse : l'extrémité de la flèche exerce en effet une zone d'ombre qui éloigne le transport sédimentaire du rivage situés à l'aval.

Ce déficit sédimentaire a engendré la morphologie actuelle de la zone occidentale de la passe lagunaire. Cette zone, correspondant à la flèche plus interne, est aujourd'hui essentiellement constituée de bas fonds, avec des structures en éventail allongées et orientées vers l'intérieur de la lagune. Le démantèlement progressif de ces morphologies déterminera l'élargissement des sections de la passe avec la diminution conséquente de l'efficacité du canal tidal, qui est déjà aujourd'hui surdimensionné par rapport à la capacité des courants tidaux.

Le brusque gradient morphologique, lié aux faibles profondeurs des fonds lagunaires et de celles élevées de la passe, ne permet pas au delta de flot de 
développer toutes ses potentialités et de s'étendre donc sur une superficie majeure. Cette morphologie, bien que représentant seulement la phase évolutive initiale de la passe, indique que l'énergie des courants tidaux se concentrera sur le côté Est du canal. De plus, le lobe occidental de la passe, où interagissent les dynamiques marines et celles lagunaires, assumera toujours un rôle plus marginal dans les processus hydrodynamiques de la zone. Le calcul des volumes mobilisés sur les fonds centre-orientaux de la passe lagunaire tend à confirmer les dynamiques évolutives décrites (Fig. 2)

Ces données sont sous-estimés puisque le calcul volumétrique ne comprend pas le développement et la consolidation des ramifications terminales du Scanno (Fig. 3), ni les sédiments du canal tidal et du delta de flot. On peut ainsi supposer que le budget obtenu doit être multiplié par un facteur de 2 ou 3 afin d'obtenir la quantité réelle du bilan sédimentaire.

Ces dernières considérations laissent supposer qu'une grande quantité de matériel puisse être prélevée pour rééquilibrer des situations côtières et lagunaires critiques. L'étude réalisée a donc permis de déterminer un dépôt de matériels sableux qui pourrait permettre de contraster la crise régressive des littoraux de l'Emilia-Romagna. De plus, il est possible de récupérer du matériel du démantèlement des ramifications plus extérieures sans compromettre la stabilité de la flèche ou l'efficacité hydraulique de la passe lagunaire.

Différents échantillons sédimentaires ont été prélevés jusqu'à une profondeur de $1.5 \mathrm{~m}$ à l'aide d'un carottier continu pour terrains cohésifs et granulaires afin de vérifier les caractéristiques texturales des matériels de dragage. Malheureusement l'étude a mis en évidence que seulement une faible quantité de sédiment pourrait être utilisée pour réaliser un éventuel rechargement des plages (SIMEONI et al., 2007a).

Toutefois il semblerait qu'un abaissement des bas fonds situés à l'extrémité de la flèche favoriserait le démantèlement des nouvelles branches terminales et, par conséquent, l'alignement des deux côtés de la passe tidale comme le confirme des simulations numériques (BRATH et DI BALDASSARRE, 2007).

La possibilité d'utiliser les sédiments prélevés sur le Scanno pour recharger les plages adjacentes (Lido di Volano et Nazioni) a été vérifiée en utilisant les méthodologies de Hobson (1977) et de l'USACE (1986, 2003). Les dimensions généralement plus fines, le contenu plus élevé de la fraction silto-argileuse des couches observées dans les carottes prélevées ont mis en évidence que seule une 
faible quantité de sédiment (généralement les premiers $30 \mathrm{~cm}$ ) pouvait être utilisée pour un éventuel rechargement. Au contraire le matériel dragué des fonds et du creusement du canal de marée pourrait être utilisé pour assainir les fonds de la lagune (activités d'élevage de coque) et pour recharger l'île barrière qui correspond au côté occidental de la passe tidale(Fig. 4).

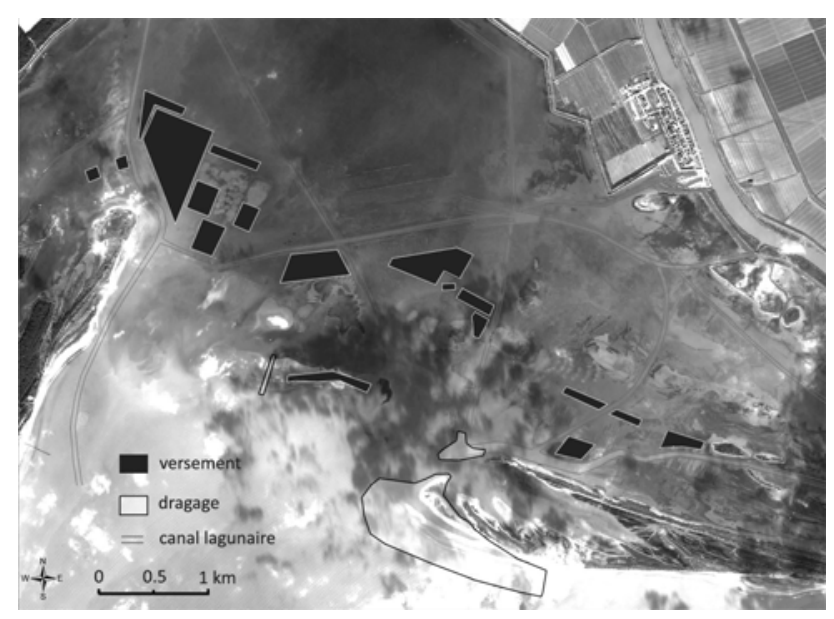

Figure 4-Représentation schématique de l'intervention proposée.

La reconstruction de l'île-barrière, aujourd'hui partiellement démantelée par la houle, engendrerait le rétrécissement de la largeur de la passe et donc une augmentation de la vitesse des courants tidaux (Fig. 5). Ceci permettrait au canal tidal de retrouver son efficacité et d'augmenter son action pour s'opposer à la dérive littorale.

Cette intervention pourrait également amorcer un phénomène de circulation secondaire d'un flux local inverse qui permettrait au système de maintenir une configuration substantiellement plus stable si il est suffisamment alimenté.

\section{Conclusions}

L'étude a été réalisée en raison de la nécessité d'intervenir sur l'hydrodynamisme de la lagune de Goro, pour sauvegarder les activités économiques, en réalisant une intervention respectueuse de l'évolution du spit et de sa valeur naturaliste, compatible avec les ressources économiques disponibles. 

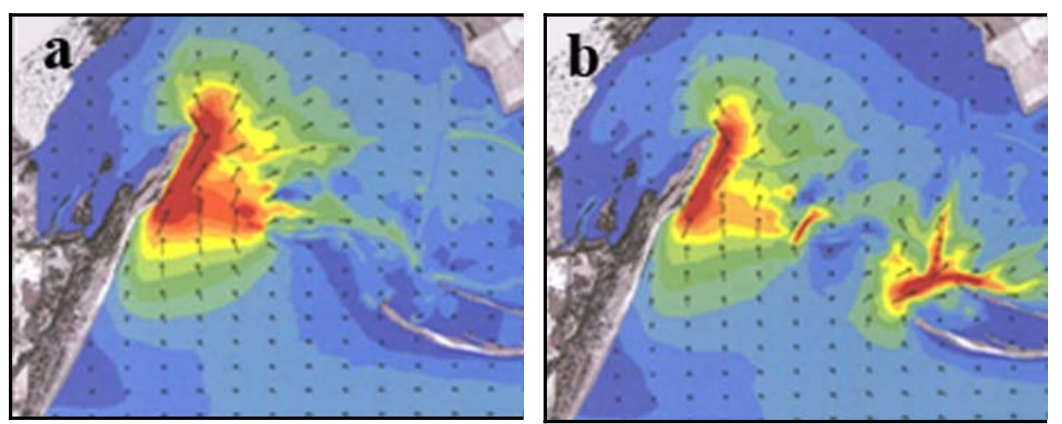

Figure 5 - Simulation de la circulation hydrique : état actuel (a) et après intervention proposée (b) (selon BRATH et DI BALDASSARRE, 2007-modifiée).

La solution choisie se base essentiellement sur le déplacement des matériels à travers le re-profilage de la plate-forme submergée de la flèche littorale et du canal de marée. Les matériels dragués plus grossiers seront versés sur l'île-barrière pour mettre en sûreté les territoires lagunaires, alors que ceux plus fins seront utilisés pour assainir les fonds lagunaires.

Cette approche de projet ne représente peut-être pas la meilleure solution technique pour rétablir l'efficacité hydraulique de la passe secondaire mais est certainement le meilleur compromis entre les exigences socio-économiques de la zone et les solutions techniques disponibles.

La réalisation du projet, déjà en cours de réalisation, a en outre prévu la coparticipation financière des coopératives de pêche, qui bénéficieront des versements sableux. Ceci ouvre de nouvelles perspectives à la gestion de la côte qui demandera toujours plus de ressources, notamment en prévision des changements climatiques prévus.

\section{Remerciements}

Nous remercions le Dott Silvano Bencivelli et le Dott. Stefano Lovo de la Province de Ferrare pour le support fourni. L'étude a été réalisée dans le cadre de la Convention pour l'Etude sur la prévision de l'évolution morphodynamique du Scanno et des Passes de la Sacca de Goro.

\section{Bibliographie}

1 BARNES R.S.K. (1980). Coastal Lagoons, Cambridge Univ. Press, Cambridge, UK, pp 106. 
2 BRATH A., DI BALDASSARE G. (2007) Studio degli effetti idrodinamici indotti nella Sacca di Goro dall'intervento di rimodellamento dello Scanno e di ripascimento delle aree in concessione mediante modellazione matematica bidimensionale. Relazione conclusiva, Provincia di Ferrara, 58p.

3 CAUMETTE P., CASTEL J., HERBERT R.A. (1996). Coastal lagoon eutrophication and anaerobic processes (C.L.E.AN). Hydrobiologia 329, $225 \mathrm{p}$.

4 GABBIANELli G., DEL GRANDE C., SIMEONI U., ZAMARIOLO A., CALDERONI G., (2000) - Evoluzione dell'area di Goro negli ultimi cinque secoli (Delta del Po). In: Umberto Simeoni (Ed), La Sacca di Goro. Studi Costieri, Firenze, 2, 45-63.

5 GIORDANI G., VIAROLI P., SWANEY D.P., MURRAY C.N., ZALDÍVAR J.M., MARSHALL CROSSLAND J. I. (2005). Nutrient fluxes in transitional zones of the Italian coast. LOICZ Reports \& Studies No 28, LOICZ, Texel, the Netherlands. $157 \mathrm{p}$

6 HOBSON, R.D., 1977 - Review of Design Elements for Beach-Fill Evaluation. TM-77-6, U.S. Army.

7 KJERFVE, B. (1994). Coastal lagoon processes. Elsevier. Amsterdam. 577 pp.

8 SIMEONI U., BRUNELLI V. (2007) - Individuazione ed ipotesi gestionali dei depositi sabbiosi della Sacca di Goro. Rapporto interno Provincia di Ferrara, 41p. 9 SIMEONI U., TESSARI U, BRUNELLI V. (2007a) - Analisi di compatabilità sedimentaria per opere di ripristino degli interscambi con il mare nella Sacca di Goro in area S.I.C. e Z.P.S. Rapporto interno Provincia di Ferrara, 32p.

10 SIMEONI U., FONTOLAN G., TESSARI U., CORBAU C. (2007b). Domains of spit evolution in the Goro area, Po Delta, Italy. Geomorphology, 86, 3-4, 332348.

11 TSIHRINTZIS V.A., SYLAIOS G.K., SIDIROPOULOU M., KOUTRAKIS E. (2007). Hydrodynamic modeling and management alternatives in a Mediterranean, fishery exploited, coastal lagoon, Aquacultural Engineering 36(3), 310-324.

12 U.S. ARMY CORPS OF ENGINEERS (2003). Beach Fill Design. EM 11102-1100 (Part V - Chapter 4): 109 p.

13 U.S. ARMY CORPS OF ENGINEERS (1986) Overfill and renourishment factors. Coastal Engineering Technical Note, CETN-II-15, 9/86. 